D.O.I.: $10.3895 / \mathrm{S} 1808-04482008000200012$

\title{
UMA ANÁLISE DAS CONEXÕES DE CONHECIMENTO ENTRE FIRMAS NA PROVÍNCIA DE PETRÓLEO E GÁS DA BACIA DE CAMPOS
}

\section{AN ANALYSIS OF KNOWLEDGE LINKAGES BETWEEN FIRMS IN THE OIL AND GAS PROVINCE OF THE CAMPOS BASIN}

\author{
Bruno dos Santos Silvestre ${ }^{1}$; Paulo Roberto Tavares Dalcol ${ }^{2}$ \\ ${ }^{1}$ Centrais Elétricas Brasileira S.A. - ELETROBRÁS - Rio de Janeiro - Brasil \\ bruno.silvestre@eletrobras.com \\ ${ }^{2}$ Pontifícia Universidade Católica do Rio de Janeiro - PUC-Rio - Rio de Janeiro - Brasil \\ prtd@,ind.puc-rio.br
}

\begin{abstract}
Resumo
O objetivo central deste trabalho consiste em verificar a relação da intensidade das conexões de conhecimento, estabelecidas entre atores para a implementação de mudanças tecnológicas na província de petróleo e gás da Bacia de Campos, e a natureza dessas conexões (intra-província e extra-província). Adota-se como hipótese a idéia de que como se trata de um arranjo de firmas atuantes em uma indústria de alta tecnologia e onde muitos dos atores mais dinâmicos são representados por subsidiárias de empresas multinacionais, as conexões de conhecimento extraprovíncia (estabelecidas com atores externos à província) sejam predominantes em número e em intensidade em relação às conexões intra-província. Para verificar esta hipótese, foram realizados dez estudos de caso em firmas situadas nesta província de petróleo e gás, e pertencentes aos grupos de fornecedores de serviços de poço e equipamentos de poço, além da própria Petrobras. A análise está centrada em duas dimenções-chave: a estrutura de conexões de conhecimento entre firmas e a intensidade dessas conexões. Com base nestas duas dimenções-chave, pôde-se identificar indícios de que as conexões extra-província são predominantes para o grupo de fornecedores de seviços de poço, mas não para o de equipamentos e para a Petrobras. No entanto, qualitativamente esta predominância não pôde ser verificada para nenhum dos grupos de firmas estudados.
\end{abstract}

Palavras-chave: Conexões de Conhecimento, Relacionamento Inter-firmas, Petróleo e Gás.

\section{Introdução}

Este trabalho consiste em parte de um estudo eminentemente empírico e concentra-se no segmento upstream de petróleo e gás situado na província da Bacia de Campos. É neste ambiente que este estudo busca inferir as interações entre variáveis complexas, tais como conexões interfirmas, conhecimento, capacitações tecnológicas, mudanças tecnológicas e inovações.

Grande parte das mudanças tecnológicas ocorridas neste segmento concentra-se no grupo de firmas que atuam no ramo de atividade caracterizado como 'fornecedores de serviços de poço' (well 
services suppliers). Este grupo é formado por empresas fornecedoras de serviços especializados em perfuração, completação de poços de petróleo, serviços de fluidos para perfuração, além da operação, manutenção e reparo dos sistemas de produção. Um outro grupo de firmas bastante dinâmico tecnologicamente, que também é objeto deste estudo são os 'fornecedores de equipamentos para cabeça de poço' (well-head equipments suppliers). Os equipamentos para cabeça de poço consistem nas estruturas e sistemas submarinos instalados na saída dos poços de produção de petróleo e gás e têm a finalidade de controlar a enorme pressão do óleo/gás expelido, direcionálo e bombeá-lo para o processamento. Tais sistemas são compostos de estrutura metálica bruta, tubos pelos quais o petróleo ou o gás irá escoar, válvulas, árvores de natal, manifolds, além de todo o sistema de controle e segurança de poço. Por esse motivo, esses dois grupos de firmas foram escolhidos como foco do trabalho de campo, além da própria Petrobras.

O objetivo central deste artigo consiste em verificar a relação entre a intensidade das conexões de conhecimento, estabelecidas com outros atores para a implementação de mudanças tecnológicas na província de petróleo e gás, e a natureza dessas conexões.

Adota-se, como hipótese, a idéia de que como se trata de um arranjo de firmas atuantes em uma indústria de alta tecnologia e onde muitos dos atores mais dinâmicos são representados por subsidiárias de empresas multinacionais, as conexões extra-província (estabelecidas com atores externos à província de petróleo e gás da Bacia de Campos) sejam predominantes em número e intensidade às conexões intra-província.

Para avaliar esta hipótese, foram realizados estudos de caso em dez firmas situadas na província de petróleo e gás da Bacia de Campos, tomando por base duas dimenções-chave: as conexões de conhecimento entre firmas estabelecidas para implementação das mudanças tecnológicas e a intensidade dessas conexões.

\section{Arcabouço Teórico}

Os estudos relativos ao relacionamento inter-firmas podem ter como foco as estruturas de produção ou o sistema de conhecimento. As estruturas de produção podem ser definidas como os projetos de produtos, materiais, máquinas, força de trabalho e conexões de transação de mercado envolvidas na produção de bens e serviços em uma dada especificação. Por outro lado, todo o estoque de conhecimento dentro das firmas e os fluxos de conhecimentos entre as firmas (conexões de conhecimento) e dentro das firmas, nos quais se baseiam as mudanças nos tipos de bens produzidos e nos métodos que são utilizados para produzi-los, é denominado de sistema de conhecimento (BELL e ALBU, 1999). 
Para esses autores, a descrição de uma firma, de um cluster, de um setor ou de uma província em termos de sua estrutura de produção, não diz muito sobre a evolução da firma, do aglomerado ou da província que se analisa, ou seja, sua história, trajetória atual ou capacidade para implementar mudanças tecnológicas no futuro. O sistema de conhecimento, por outro lado, "engloba os fluxos de conhecimento, estoques de conhecimento e sistemas organizacionais envolvidos em gerar e gerir mudanças em produtos, processos e organização da produção" (BELL e ALBU, 1999: 1723).

A importância do conhecimento e, conseqüentemente, das conexões de conhecimento, não parece ser contestada na literatura. É necessário ressaltar que tão importante quanto a existência de um sistema de conhecimento robusto é a capacidade de absorção desse conhecimento pelas firmas (MALERBA e ORSENIGO, 2000; DAHL e PEDERSEN, 2001; ENGEN e OLSEN, 2004; GIULIANI, 2004). Por esses motivos este estudo apresenta um viés focado no sistema de conhecimento e não na estrutura de produção da província de petróleo e gás da Bacia de Campos.

As conexões de conhecimento podem ser intra-província (estabelecida com um ator localizado dentro da própria província) ou extra-província (estabelecida com um ator localizado fora da província), conforme Quadro 1. A importância das conexões tanto intra quanto extraprovíncia, para o processo de absorção do conhecimento e desenvolvimento de capacitaçõies tecnológicas, é bastante discutida e reconhecida na literatura da área (KIM e TUNZELMANN, 1998; BELL e ALBU, 1999; LANE, 2001; CIARLI, 2003).

\section{Quadro 1: Natureza das Conexões de Conhecimento}

\begin{tabular}{|c|c|c|c|}
\hline \multicolumn{2}{|c|}{ Estrutura de Conexões } & Aberta & \multicolumn{2}{|c|}{ Externamente } \\
\cline { 2 - 4 } Internamente & Conectada & $\begin{array}{c}\text { Estrutura conectada com atores do ambiente interno } \\
\text { à aglomeração econômica e conectada com atores } \\
\text { do ambiente externo à aglomeração (estrutura } \\
\text { aberta) }\end{array}$ & $\begin{array}{c}\text { Estrutura conectada com atores do ambiente interno à } \\
\text { aglomeração econômica e não-conectada com atores } \\
\text { do ambiente externo à aglomeração (estrutura } \\
\text { fechada) }\end{array}$ \\
\cline { 2 - 4 } & $\begin{array}{c}\text { Não- } \\
\text { Conectada }\end{array}$ & $\begin{array}{c}\text { Estrutura não-conectada com atores do ambiente } \\
\text { interno à aglomeração econômica e conectada com } \\
\text { atores do ambiente externo à aglomeração (estrutura } \\
\text { aberta) }\end{array}$ & $\begin{array}{c}\text { Estrutura não-conectada com atores do ambiente } \\
\text { interno à aglomeração econômica e não-conectada } \\
\text { com atores do ambiente externo à aglomeração } \\
\text { (estrutura fechada) }\end{array}$ \\
\hline
\end{tabular}

FONTE: Silvestre (2006)

Além disso, essas conexões podem ainda ser classificadas de acordo com suas intensidades, conforme estudos encontrados na literatura, tais como Athreye, 2001; Baldwin e Hanel, 2003 (Quadro 2). 
Quadro 2: Intensidade das Conexões de Conhecimento

\begin{tabular}{|c|c|}
\hline GRAU DE INTENSIDADE & CARACTERÍSTICAS \\
\hline FORTE & $\begin{array}{l}\text { Conexão relacionada com estabelecimento de instrumentos de cooperação formais em P\&D (contratos). O estabelecimento deste tipo } \\
\text { de conexão demonstra um esforço deliberado para dominar a tecnologia em questão. A aquisição de conhecimento e capacitações } \\
\text { tecnológicas é intensa. }\end{array}$ \\
\hline MODERADA & $\begin{array}{l}\text { Conexão relacionada com políticas de treinamento de técnicos e engenheiros no Brasil e no exterior, incorporando as capacitações } \\
\text { tecnológicas à base de conhecimento da firma. O estabelecimento deste tipo de conexão demonstra uma postura mais ativa do que } \\
\text { passiva da firma. A aquisição de conhecimento e capacitação tecnológicas é moderada. }\end{array}$ \\
\hline FRACA & $\begin{array}{l}\text { Conexão relacionada com a contratação de consultorias técnicas especializadas, tanto nacionais como estrangeiras. O } \\
\text { estabelecimento deste tipo de conexão demonstra uma postura mais passiva do que ativa. A aquisição de conhecimento e } \\
\text { capacitações tecnológicas é pequena. }\end{array}$ \\
\hline MUITO FRACA & $\begin{array}{l}\text { Conexão relacionada com a troca de informações e conhecimento tecnológico informalmente. O estabelecimento deste tipo de } \\
\text { conexão demostra uma postura passiva, sem o esforço deliberado da firma em incorporar esse conhecimento de forma sistemática. A } \\
\text { aquisição de conhecimento e capacitações tecnológicas é muito pequena. }\end{array}$ \\
\hline
\end{tabular}

FONTE: Silvestre (2006)

Para Viotti (2002; 2003), as firmas apresentam posturas tecnológicas que podem ser ativas ou passivas, de acordo com os tipos de conexões encontrados. A postura de absorção ativa está baseada no esforço deliberado em entender e dominar o fluxo de conhecimento recebido, por meio de investimento de dinheiro e de tempo (conexões de intensidades fortes e moderadas). Na forma passiva, o esforço para entender e dominar o fluxo de conhecimento recebido é mínimo e a absorção é automática e pouco custosa (conexões de intensidades fracas e muito fracas).

O termo capacitação tecnológica é definido, para efeito deste trabalho, no sentido utilizado por Figueiredo (2003: 38), como os "recursos necessários para gerar e administrar mudanças tecnológicas, tais como aptidões, conhecimento e experiência, e sistemas organizacionais”. Do mesmo modo, utiliza-se uma desagregação de dois tipos diferentes de capacitações tecnológicas, conforme utilizado pelo autor na indústria siderúrgica nacional. Essa desagregação faz a distinção entre as 'capacitações tecnológicas rotineiras', conceituadas como as aptidões necessárias para usar a tecnologia, o conhecimento e os mecanismos organizacionais, e as 'capacitações tecnológicas inovadoras', que consistem em aptidões que permitem criar, modificar, ou aperfeiçoar produtos e processos.

\section{Metodologia}

Dez estudos de caso foram realizados na província de petróleo e gás da Bacia de Campos. A unidade de coleta de dados consistiu nas mudanças tecnológicas implementadas por essas firmas na província. Essa estratégia foi adotada no sentido de fornecer praticidade na referida fase. Na ocasião das entrevistas o foco foi direcionado para eventos tecnológicos específicos (mudanças tecnológicas implementadas) e não para a firma como um todo, conforme estratégia usada por Baldwin e Hanel (2003). Os eventos foram identificados pelo próprio entrevistado (informante-chave), representando, portanto, as principais mudanças tecnológicas implementadas nos últimos anos por aquela empresa na província. Na medida do possível, a importância dos eventos foi checada com 
outros funcionários das próprias firmas, com suas concorrentes e com a Petrobras em entrevista subseqüente. Tal estratégia ajudou a atenuar alguns problemas identificados antes do trabalho empírico: o pequeno número de empresas em cada grupo e o receio de perder o foco no momento das entrevistas. Com essa estratégia pôde-se aumentar o número de pontos de análise (por meio dos eventos) e direcionar a entrevista para um único evento, o que aumentou a precisão das respostas. Essa estratégia permitiu, portnato, que os eventos fossem em número bem superior ao número de firmas inseridas no trabalho empírico.

A eficácia da estratégia utilizada fica mais evidente na medida em que se verifica que foram realizadas entrevistas em dez (10) firmas, estabelecendo vinte e cinco (25) eventos válidos no total, sendo que cada uma das firmas identificou entre um (1) e quatro (4) eventos tecnológicos. Nos vinte e cinco (25) eventos válidos, setenta e cinco (75) conexões de conhecimento foram identificadas, sendo que cada um deles apresentou de uma (1) a cinco (5) conexões diferentes.

Em relação às conexões de conhecimento identificadas (primeira dimensão-chave), estas foram classificadas nas seguintes categorias: intra-província e extra-província. As conexões de conhecimento intra-província são as conexões estabelecidas entre as firmas estudadas e atores localizados dentro da própria província de petróleo e gás da Bacia de Campos. As conexões de conhecimento extra-província são as conexões establecidas entre as firmas pesquisadas e atores localizados fora da província de petróleo e gás da Bacia de Campos (podendo estar localizados dentro do Estado do Rio de Janeiro, do Brasil ou até mesmo no exterior).

Além disso, cada tipo de conexão de conhecimento foi classificado de acordo com o grau de intensidade apresentado (segunda dimensão-chave). Conexões de conhecimento em que o fluxo é executado apenas por meio de troca de informações (TI) foram classificadas como um grau de intensidade 'muito fraco'; conexões em que o fluxo acontece por meio de consultoria técnica (CT) foram classificadas como um grau de intensidade 'fraco'; conexões onde os fluxos são verificados por meio de treinamento de mão-de-obra (TR) foram classificadas como um grau 'moderado' de intensidade; e conexões de $\mathrm{P} \& \mathrm{D}$, estabelecidas por meio de instrumentos formais (contratos) foram classificadas como grau de intensidade 'forte' (PD).

Nesse sentido, caso a hipótese estabelecida anteriormente seja aceita, será confirmada a predominância das conexões de conhecimento extra-província, em relação às conexões intraprovíncia (quantitativa e qualitativamente), para a absorção de conhecimento e capacitações tecnológicas das firmas, aspectos estes fundamentais para a implementação de mudanças tecnológicas e inovações. 


\section{Resultados}

No grupo de fornecedores de serviços de poço, identificou-se um total de vinte e oito (28) conexões de conhecimento nos doze (12) eventos tecnológicos válidos, perfazendo uma média de 2,33 conexões de conhecimento para cada evento.

Outra consideração importante a respeito desse grupo, e que pode ser verificada no Quadro 3 , consiste no fato de que $28,6 \%$ das conexões de conhecimento verificadas terem sido estabelecidas com atores dentro da província. As conexões de conhecimento intra-província apresentadas por este grupo foram estabelecidas integralmente com unidades da Petrobrás ou do CENPES localizados na própria região.

As conexões de conhecimento extra-província representaram $71,4 \%$ das conexões deste grupo. Destas conexões extra-província, $60,7 \%$ foram estabelecidas com a matriz da subsidiária no Brasil ou no exterior.

Quadro 3: Natureza das Conexões X Atores - Fornecedores de Serviço
\begin{tabular}{|l|c|c|c|}
\hline & Intra-província & Extra-província & TOTAL \\
\hline Petrobras/CENPES & $28,6 \%$ & & $\mathbf{2 8 , 6 \%}$ \\
\hline Fornecedores & & $7,1 \%$ & $\mathbf{7 , 1} \%$ \\
\hline Consumidores & & $3,6 \%$ & $\mathbf{3 , 6 \%}$ \\
\hline Matriz & & $60,7 \%$ & $\mathbf{6 0 , 7} \%$ \\
\hline Outras & & & $\mathbf{0 , 0} \%$ \\
\hline TOTAL & $\mathbf{2 8 , 6 \%}$ & $\mathbf{7 1 , 4 \%}$ & $\mathbf{1 0 0 , 0} \%$ \\
\hline
\end{tabular}

FONTE: Estudo Empírico

No entanto, do total de conexões, $17,9 \%$ apresentam intensidade classificada como forte ou moderada (somatório de 14,3\% e 3,6\%) e estabelecidas com atores localizados dentro da província. Muito mais significativo consiste os $35,7 \%$ das conexões de conhecimento com intensidades fortes e moderadas (somatório de 14,3\% e 21,4\%) estabelecidas com atores localizados fora da província, conforme Quadro 4.

Quadro 4: Natureza das Conexões X Intensidades - Fornecedores de Serviços de Poço

\begin{tabular}{|l|c|c|c|}
\hline & Intra-província & Extra-província & TOTAL \\
\hline FORTE & $14,3 \%$ & $14,3 \%$ & $\mathbf{2 8 , 6 \%}$ \\
\hline MODERADA & $3,6 \%$ & $21,4 \%$ & $\mathbf{2 5 , 0} \%$ \\
\hline FRACA & $10,7 \%$ & $35,7 \%$ & $\mathbf{4 6 , 4 \%}$ \\
\hline MUITO FRACA & & & $\mathbf{0 , 0} \%$ \\
\hline \multicolumn{1}{|c|}{ TOTAL } & $\mathbf{2 8 , 6 \%}$ & $\mathbf{7 1 , 4 \%}$ & $\mathbf{1 0 0 , 0 \%}$ \\
\hline
\end{tabular}

FONTE: Estudo Empírico 
Essas considerações mostram que as firmas fornecedoras de serviços de poço, nos eventos estudados, apresentam conexões de conhecimento mais numerosas com atores localizados fora da província. No entanto, em termos qualitativos, as conexões intra e extra-província parecem se equilibrar entre intensidades fortes/moderadas e fracas/muito fracas.

Além disso, ainda de acordo com o Quadro 4, apenas 28,6\% do total das conexões identificadas no grupo de fornecedores de serviço de poço foram classificadas como fortes. As intra-província com a Petrobras ou CENPES e as extra-províncias com suas matrizes localizadas no Brasil ou no exterior.

No grupo de fornecedores de equipamentos de poço, identificou-se um total de trinta e uma (31) conexões de conhecimento nos nove (9) eventos tecnológicos válidos, perfazendo uma média de 3,44 conexões de conhecimento para cada evento.

De acordo com os dados coletados no estudo empírico, verificou-se, conforme Quadro 5, que $51,6 \%$ do total de conexões de conhecimento verificadas nos eventos tecnológicos são estabelecidas com atores dentro da própria província, enquanto os outros 48,4\%, foram estabelecidos com atores externos à província. Destas conexões extra-província, 45,2\% foram estabelecidas com outras unidades da empresa (ou matriz ou outra subsidiária), localizadas no Brasil ou no exterior.

Quadro 5: Natureza das Conexões X Atores - Fornecedores de Equipamentos de Poço

\begin{tabular}{|l|c|c|c|}
\hline & Intra-província & Extra-província & TOTAL \\
\hline Petrobras/CENPES & $51,6 \%$ & & $\mathbf{5 1 , 6 \%}$ \\
\hline Fornecedores & & $3,2 \%$ & $\mathbf{3 , 2} \%$ \\
\hline Consumidores & & & $\mathbf{0 , 0} \%$ \\
\hline Matriz & & $45,2 \%$ & $\mathbf{4 5 , 2} \%$ \\
\hline Outras & & & $\mathbf{0 , 0} \%$ \\
\hline \multicolumn{1}{|c|}{ TOTAL } & $\mathbf{5 1 , 6 \%}$ & $\mathbf{4 8 , 4 \%}$ & $\mathbf{1 0 0 , 0} \%$ \\
\hline
\end{tabular}

FONTE: Estudo Empírico

Do total de conexões, 51,6\% apresentam intensidade classificada como forte ou moderada e estabelecidas com atores localizados dentro da província. Diferentemente do grupo de fornecedores de serviços de poço, apenas 3,2\% das conexões de conhecimento com intensidades fortes e moderadas foram estabelecidas com atores localizados fora da província, conforme Quadro 6. 
Quadro 6: Natureza das Conexões X Intensidades - Fornecedores de Equipamentos de Poço

\begin{tabular}{|l|c|c|c|}
\hline & Intra-província & Extra-província & TOTAL \\
\hline FORTE & $51,6 \%$ & $3,2 \%$ & $\mathbf{5 4 , 8} \%$ \\
\hline MODERADA & & & $\mathbf{0 , 0} \%$ \\
\hline FRACA & & $9,7 \%$ & $\mathbf{9 , 7} \%$ \\
\hline MUITO FRACA & & $35,5 \%$ & $\mathbf{3 5 , 5 \%}$ \\
\hline \multicolumn{1}{|c|}{ TOTAL } & $\mathbf{5 1 , 6 \%}$ & $\mathbf{4 8 , 4 \%}$ & $\mathbf{1 0 0 , 0} \%$ \\
\hline
\end{tabular}

FONTE: Estudo Empírico

Essas considerações mostram que as firmas fornecedoras de equipamentos de poço, nos eventos estudados, apresentam conexões de conhecimento ligeiramente mais numerosas com atores localizados dentro da província. Em termos qualitativos, as conexões intra-província mostraram um predomínio significativo para as intensidades fortes/moderadas em relação às intensidades fracas/muito fracas (Quadro 6).

Cabe ressaltar que identificou-se quatro (4) eventos tecnológicos relacionados à Petrobras por meio de entrevistas com informantes-chave da própria operadora (gerentes da base da Bacia de Campos). Neste eventos foram identificadas dezesseis (16) conexões de conhecimento, perfazendo uma média de 4,0 conexões por evento. É interessante destacar que todas as conexões identificadas para a implementação de mudanças tecnológicas pela Petrobras possuíam a totalidade das conexões primárias estabelecidas com atores localizados na própria província.

A Petrobrás utiliza, predominantemente, capacitações tecnológicas inovadoras em dois (2) dos eventos identificados no trabalho de campo. Esses eventos, onde a Petrobrás possui maior domínio da tecnologia, consistem nas mudanças tecnológicas relacionadas a equipamentos de poço. Nesses eventos foi identificada uma postura mais ativa da Petrobras, enquanto em outros dois (relacionados a eventos de serviços de poço), a operadora apresentou postura mais passiva com a utilização predominante de capacitações tecnológicas rotineiras.

A análise das intensidades das conexões revelou ainda que os fornecedores de serviços de poço apresentaram tendência a estabelecer conexões de conhecimento fortes e moderadas quase que na mesma proporção que conexões fracas e muito fracas (Quadro 4). No entanto, as conexões extraprovíncia possuem uma predominância significativa em relação às conexões intra-província. Já os fornecedores de equipamentos de poço apresentaram maior número de conexões fortes e moderadas (o que indica uma postura mais ativa em relação à absorção da tecnologia), sendo que cerca da metade delas foi estabelecida com atores localizados dentro da própria província, o que mostra a importância da concentração geográfica para as atividades inovadoras das firmas deste grupo (Quadro 6). Em relação à Petrobras, o predomínio das conexões de conhecimento intra-província é notório, sendo que a maioria delas apresenta intensidades fortes ou moderadas. 


\section{Considerações Finais}

De uma forma geral, as firmas fornecedoras de equipamentos de poço, com fábricas localizadas no Brasil (o que proporciona maior nível de absorção de capacitações tecnológicas inovadoras) e conexões de conhecimento de intensidade forte com atores localizados na própria província de petróleo e gás, apresentaram uma postura mais ativa e mais relacionada à utilização de capacitações tecnológicas inovadoras. De forma contrária, as firmas fornecedoras de serviços de poço com poucas conexões com atores localizados na província, apresentam postura mais passiva, relacionada à execução de tarefas, cujas tecnologias são, na grande maioria das vezes, coordenadas por suas matrizes e centros de pesquisa no exterior.

Essa situação é reflexo, em grande parte, da própria postura da Petrobras em relação a essas duas atividades. Nas atividades relacionadas a equipamentos de poço, a Petrobras apresenta uma postura ativa, direcionando, coordenando e exigindo melhorias e mudanças tecnológicas direcionadas para o contexto operacional da Bacia de Campos. Essa situação faz com que as firmas fornecedoras de equipamentos de poço desenvolvam as capacitações tecnológicas inovadoras para se relacionar, no assunto tecnologia, com seu cliente. O mesmo não acontece com as firmas fornecedoras de serviços de poço, área em que a Petrobras apresenta uma postura mais passiva, deixando o serviço a cargo das próprias contratadas.

Voltando a hipótese estabelecida no início do artigo, para a Petrobras, verificou-se uma predominância quantitativa total das conexões de conhecimneto intra-província (dezesseis conexões intra e nenhuma extra-província) - Quadro 7, mas um equilíbrio na parte qualitativa (entre intensidade forte/moderada e fraca/muito fraca), conforme Quadro 8.

Quadro 7: Natureza das Conexões X Grupos de Firmas

\begin{tabular}{|l|c|c|c|}
\hline & Intra-província & Extra-província & TOTAL \\
\hline Petrobras & 16 & 0 & 16 \\
\hline Equipamentos & 16 & 15 & 31 \\
\hline Serviços & 8 & 20 & $\mathbf{2 8}$ \\
\hline \multicolumn{1}{|c|}{ TOTAL } & $\mathbf{4 0}$ & $\mathbf{3 5}$ & $\mathbf{7 5}$ \\
\hline
\end{tabular}

FONTE: Estudo Empírico

Para os fornecedores de equipamentos de poço, verificou-se um equilíbrio quantitativo das conexões de conhecimneto extra e intra-província (dezesseis conexões intra e quinze extraprovíncia) - Quadro 7, e uma pequena predominância qualitativa para as conexões de intensidade 
forte/moderada (dezessete conexões classificadas como forte/moderada e catorze fraca/muito fraca), conforme Quadro 8.

Quadro 8: Intensidade das Conexões X Grupos de Firmas

\begin{tabular}{|l|c|c|c|}
\hline & $\begin{array}{c}\text { Forte/ } \\
\text { Moderada }\end{array}$ & $\begin{array}{c}\text { Fraca/ } \\
\text { Muito Fraca }\end{array}$ & TOTAL \\
\hline Petrobras & 8 & 8 & $\mathbf{1 6}$ \\
\hline Equipamentos & 17 & 14 & $\mathbf{3 1}$ \\
\hline Serviços & 15 & 13 & $\mathbf{2 8}$ \\
\hline \multicolumn{1}{|c|}{ TOTAL } & $\mathbf{4 0}$ & $\mathbf{3 5}$ & $\mathbf{7 5}$ \\
\hline
\end{tabular}

FONTE: Estudo Empírico

Para os fornecedores de serviço de poço, verificou-se uma predominância quantitativa das conexões de conhecimneto extra-província (vinte conexões extra e oito intra-província) - Quadro 7, e uma pequena predominância na parte qualitativa para as conexões de intensidade forte/moderada (quinze conexões classificadas como forte/moderada e treze fraca/muito fraca), conforme Quadro 8.

Em relação a totalidade das firmas (fornecedores de equipamentos, serviços e Petrobras) não se pode afirmar que a idéia de que um arranjo de firmas relacionado a uma indústria de alta tecnologia e onde muitos dos atores mais dinâmicos são representados por subsidiárias de empresas multinacionais, signifique uma predominância quantitativa das conexões de conhecimento extraprovíncia. Da mesma forma, qualitativamente (intensidade), as conexões de conhecimento extraprovíncia não parecem ser predominantes às intra-província.

Isso implica em rejeitar a hipótese estabelecida inicialmente e indica que a proximidade das firmas na província de petróleo e gás da Bacia de Campos é importante para o seu relacionamento e para o estabelecimento de conexões de conhecimento.

\begin{abstract}
The central goal of this paper is to verify the relation between the knowledge linkages intensity, implemented between actors to carry out technological changes in the oil and gas province of the Campos Basin, and the nature of these linkages (intra-province and extra-province). We adopt as hypothesis the idea that as an industrial agglomeration that act in a high technology industry and where many of the most dynamics actors are multinational companies (or their subsidiaries), the extra-province linkages (implemented with actors from outside the province) are dominant in number and in intensity in relation to the intra-province linkages. To verify that hypothesis, we carried out ten case studies in firms that are located inside the oil and gas province, and belonging to the well services and well equipments suppliers group, and also with Petrobras. The analysis is centered in two key-dimensions: the knowledge linkages structure and the intensity of these linkages. Based on these two key-dimensions, we could identify some evidences that the extra-
\end{abstract}


province linkages are predominant for the well services suppliers group, but not for the well equipment suppliers group and neither for Petrobras. However, qualitatively this predominance could not be verified to none of the studied groups.

Key-Words: Knowledge Linkages, Inter-firms Relationship, Oil and Gas.

\section{Referências}

ATHREYE, S. S.; Agglomeration and Growth: A Study of the Cambridge Hi-Tech Cluster. Stanford Institute for Economic Policy Research, SIEPR Discussion Paper No. 00-42, 2001.

BALDWIN, J. R.; HANEL, P. Innovation and Knowledge Creation in an Open Economy: Canadian Industry and International Implications. Cambridge University Press, Cambridge, 2003.

\section{cross ${ }^{\text {ref }}$}

BELL, R. M.; ALBU, M. Knowledge Systems and Technological Dynamism in Industrial Clusters in Developing Countries. World Development 27 (9): 1715. 1722-3.1726 1914, 1999.

CIARLI, T. Innovation Systems and Industrialisation Processes: An Agent Based Model to Explain Local Emergence through Internal and External Relations. DRUID Summer Conference, 2003.

DAHL, S. M.; PEDERSEN, C. O. R. Knowledge Flows through Informal Contacts in Industrial Clusters: Myths or Realities? DRUID Working Paper n $n^{\circ}$ 03-01, 2001.

ENGEN, O. A.; OLSEN, O. E. Stability and Change in Industrial Knowledge Networks. Paper presented on DRUID Summer Conference, 2004.

FIGUEIREDO, P. N. Aprendizagem Tecnológica e Performance Competitiva. Ed. Fundação Getúlio Vargas. Rio de Janeiro, 2003.

GIULIANI, E. When the micro shapes the meso: learning and innovation in wine clusters. DPhil Thesis, SPRU, University of Sussex, 2004.

KIM, S. R. e TUNZELMANN, N. V. Aligning internal and external networks: Taiwan's specialization in IT. SPRU Electronic Working Paper Series 17, 1998.

LANE, D. A. Complexity and Local Interactions: Towards a Theory of Industrial Districts. Mountedison Foundation, Milão, 2001.

MALERBA, F.; ORSENIGO, L. Knowledge, innovative activities and industry evolution. Industrial and Corporate Change, Vol. 9, $\mathrm{n}^{\circ}$ 2, 2000.

SILVEStre, B. S. Aglomeração Industrial de Petróleo e Gás da Região Produtora da Bacia de Campos: Conexões de Conhecimento e Posturas Tecnológicas das Firmas. Tese de Doutorado, Departamento de Engenharia Industrial (DEI), PUC-Rio, 2006.

VIOTTI, E. B. National Learning Systems - A new approach on technological change in late industrializing economies and evidences from the cases of Brazil and South Korea Technological Forecasting and Social Change, No 69, pp. 653-680, 2002.

VIOTTI, E. B. Technological Learning Systems, Competitiveness and Development. Paper presented on First Globelics Conference - Innovation Systems and Development Strategies for the Third Millennium, Rio de Janeiro, 2003. 


\section{Dados completos de TODOS os autores:}

Nome completo: Bruno dos Santos Silvestre

Filiação institucional: Centrais Elétricas Brasileiras S. A. - ELETROBRÁS

Departamento: Departamento de Desenvolvimento de Negócios - DFN

Função ou cargo ocupado: Supervisor de Negócios

Endereço completo para correspondência (bairro, cidade, estado, país e CEP): Rua Álvaro Tâmega - 29, Centro, Campos dos Goytacazes - RJ, CEP: 28035-030; Brasil.

Telefones para contato: (22) 9811-6645; (21) 2514-6064

e-mail: bruno.silvestre@eletrobras.com ou bruss@ind.puc-rio.br

Nome completo: Paulo Roberto Tavares Dalcol

Filiação institucional: Pontifícia Universidade Católica do Rio de Janeiro - PUC-Rio

Departamento: Departamento de Engenharia Industrial - DEI

Função ou cargo ocupado: Professor Associado

Endereço completo para correspondência (bairro, cidade, estado, país e CEP): Rua Marquês de São Vicente, 225 - Gávea, Rio de Janeiro, RJ, CEP: 22453-900

Telefones para contato: (21) 3527-1284, 3527-1285

e-mail:prtd@ind.puc-rio.br 